\title{
¿LAS CORRELACIONES ALTMÉTRICAS/MÉTRICAS TRADICIONALES SE VEN AFECTADAS POR LOS PLANES DE MARKETING EN MEDIOS SOCIALES DE LAS REVISTAS? EL CASO DE LA REVISTA COMUNICAR
}

\author{
Nieves González-Fernández-Villavicencio* \\ Biblioteca de la Facultad de Económicas y Empresariales. Universidad de Sevilla. \\ Víctor Manuel Moya-Orozco** \\ Biblioteca de la Facultad de Educación. Universidad de Sevilla.
}

\begin{abstract}
Resumen: En este estudio se analizan las correlaciones entre los datos aportados por los sistemas de citas tradicionales y las altmétricas de los artículos publicados durante cinco años en la revista Comunicar, que destaca sobre las demás de su área por su avanzado plan de marketing en medios sociales. Además, se presentan los indicadores que ofrecen datos altmétricos y se lleva a cabo una revisión bibliográfica sobre las publicaciones que han tratado el tema de las correlaciones, destacando relaciones débiles entre métricas tradicionales y altmétricas. Del estudio de correlaciones sobre los datos de la revista, se confirman las débiles relaciones entre los tipos de métricas y se concluye que son significativas para evaluar el nivel de atención que reciben las publicaciones en distintos ámbitos. Como recomendación a los investigadores y editores se destaca la importancia de utilizar los medios sociales, en especial Mendeley y Twitter, para dar visibilidad y difundir la producción científica.

Palabras clave: Comunicación científica; correlaciones; web social; altmétricas; Snowball; Mendeley; Twitter;
\end{abstract} Altmetric.com.

Title: ARE ALTMETRICS-TRADITIONAL SYSTEMS CORRELATIONS AFFECTED BY THE JOURNALS' SOCIAL MEDIA MARKETING PLAN?: THE JOURNAL COMUNICAR.

Abstract: This study analyses the correlations between data provided by the traditional citation systems on one hand and altmetrics on the other hand, related to the articles published in the journal Comunicar during five years. This journal stands out among the others in its field due to its advanced social media marketing plan. First, a presentation of the most representative aspects of altmetrics is given, according to published studies. Next, a bibliographic review is carried out on the publications dealing with the subject of the correlations and that emphasize the low correlation between citations and altmetrics. With the aim of helping researchers to understand what they involved and how they are used for their own benefit, this review is followed by a cross-metric validation study of the articles that concludes that altmetrics do not measure the authors' scientific impact, but they are significant in assessing the level of attention received by their publications in different fields and indicate the interest and the importance of using social media, especially Mendeley and Twitter, to give visibility and disseminate their scientific outputs.

Keywords: Scientific communication; correlations; Social Web; altmetrics; Altmetric.com; Snowball; Mendeley; Twitter.

Copyright: (C) 2018 Servicio de Publicaciones de la Universidad de Murcia (Spain). Este es un artículo de acceso abierto distribuido bajo los términos de la licencia Creative Commons Reconocimiento 4.0 Internacional (CC BY $4.0)$.

\section{INTRODUCCIÓN Y ESTADO DE LA CUESTIÓN}

Desde que en 2010 fueran propuestas en Altmetrics: a manifesto (Priem et al., 2010), las altmétricas han generado diversas expectativas en el mundo académico, pero también preguntas sobre su capacidad real para evaluar y medir el impacto de la producción científica. En trabajos más recientes se van conociendo experiencias de uso (Martín-Martín et al., 2016), al mismo tiempo que se despejan algunas de las incógnitas de los primeros momentos (Erdt et al., 2016).

Entre sus ventajas destaca la de ofrecer indicadores sobre resultados de investigación más allá del artículo científico. Diversas instituciones, como la National Science Foundation (Piwowar, 2013), permiten incluir en sus solicitudes de financiación otros tipos de resultados de investigación diferentes al artículo, ampliando la variedad documental que evalúan. No obstante, la ausencia de identificadores únicos o estables para estas otras evidencias -

\footnotetext{
*nievesg@us.es

**vmoya@us.es
}

Recibido: 31-08-2017; $2^{\mathrm{a}}$ versión: 22-12-2017; aceptado: 04-01-2018.

GONZÁLEZ FERNÁNDEZ-VILLAVICENCIO, N. y MOYA OROZCO, V.M. ¿Las correlaciones altmétricas/métricas tradicionales se ven afectadas por los planes de marketing en medios sociales de las revistas? El caso de la revista Comunicar. Anales de Documentación, 2018, vol. 21, nº 1. Disponible en: http://dx.doi.org/10.6018/analesdoc.21.1.303221. 
documentos oficiales o menciones en noticias-, impide que los agregadores altmétricos las rastreen y analicen en las plataformas sociales.

Entre las cuestiones más debatidas se encuentra su capacidad para medir el impacto de la producción científica, es decir, en la capacidad que el autor, artículo o revista tiene para influir en la dirección, desarrollo o progreso en su campo de investigación. Ese impacto facilita las posibilidades de ser descargado, leído o posteriormente citado, pero no habla necesariamente de la calidad del artículo (Patton, Stahl y Wells, 2016). Bornmann y Haunschild (2016b) señalan el impacto de la investigación en todos los sectores de la sociedad-económico, cultural o político-y la NISO National Information Standards Organization, en su estudio para normalizar estas métricas, es consciente de que la naturaleza del impacto científico es diferente para cada grupo de interés (NISO, 2016).

Los estudios realizados, así como la propia práctica, han demostrado que las altmétricas no ofrecen un dibujo completo del impacto académico como tampoco lo hacen las métricas tradicionales (Bornmann, 2014; Konkiel, 2016; González-Fernández-Villavicencio, 2016). El debate gira en torno a qué miden exactamente estos datos y cómo aplicarlos a la evaluación científica (Sugimoto et al., 2017). El consenso llega por su carácter complementario a las métricas tradicionales (Torres-Salinas, Cabezas-Clavijo y Jiménez-Contreras, 2013; Costas, Zahedi y Wouters, 2015a), pues ayudan a completar el alcance de la investigación más allá de los canales tradicionales y ofrecen «indicadores de impacto rápidos y en tiempo real, públicamente accesibles y transparentes, incluyen una amplia audiencia no académica y cubren una mayor diversidad de productos y fuentes de investigación» (Erdt et al., 2016). Para la NISO (2016), aunque estas métricas no son válidas para una evaluación científica alternativa, se distinguen de las tradicionales basadas en citas por la inclusión de diferentes productos de investigación y formas de interacción para dejar abierta la posibilidad de un uso complementario.

No obstante, la mayor parte de los estudios se han centrado en la validación de métricas cruzadas, estudiando la correlación entre indicadores altmétricos y bibliométricos tradicionales que han demostrado la baja relación entre ambos sistemas, excepto cuando los cálculos se realizan con Mendeley (Erdt et al., 2016) y con Twitter (Hassan y Gillani, 2016).

Con respecto a su uso, han ido apareciendo recolectores de datos altmétricos, siendo Altmetric.com el que ha recibido mayor atención hasta el momento (Erdt et al., 2016). Algunos de estos recolectores pertenecen o están ligados a grandes grupos editoriales (Plum Analytics y Snowball a Elsevier o ImpactStory a Nature y más recientemente a Clarivate Analytics) y cada uno establece qué datos recoge y cómo lo hace, de manera que contrastando los datos entre plataformas es frecuente encontrar diferencias en los resultados (Erdt et al., 2016).

Altmetric.com, uno de los primeros agregadores de indicadores altmétricos, ofrece datos que obtiene de diferentes plataformas y los combina para crear el Altmetric Attention Score (AAS), métrica que permite comparar la atención recibida por un artículo con otros similares.

Snowball es una iniciativa de colaboración entre el ámbito académico y el comercial (Elsevier) que ofrece indicadores para la evaluación de la calidad de la investigación mediante una variedad de datos y no sólo de citas (Patton et al., 2016). Reutiliza las altmétricas de Altmetric.com, Plum Analytics e ImpactStory (Erdt et al., 2016, p. 1121) y su objetivo es convertirse en un estándar para el benchmarking institucional y cubrir el espectro de las actividades de investigación. En 2017, Elsevier adquiere Plum Analytics y Clarivate Analytics se asocia con Impactstory, enriqueciendo los datos altmétricos de sus registros y consolidando el papel que están jugando el acceso abierto y las altmétricas en la difusión y valoración de la producción científica.

Las revistas científicas son cada vez más conscientes de la importancia que tiene la promoción de sus publicaciones en los medios sociales, cuyo resultado esperado es un aumento de las puntuaciones altmétricas. En el trabajo de González-Fernández-Villavicencio (2017) se plantea una clasificación de las revistas en función de su política de marketing en medios sociales, destacando aquellas que tienen su propia política de promoción y cuentan con una batería de recomendaciones que destacan en su página web. Este es el caso, único en España, de la revista Comunicar. En la normativa para autores ${ }^{1}$ aparece expresamente un conjunto de indicaciones para la promoción y difusión del artículo, comprometiendo a estos a participar activamente en las redes sociales de la revista y animándoles tanto a la promoción como a la difusión de los trabajos en sus diversas versiones, en redes sociales generalistas y científicas, webs personales o institucionales, repositorios y gestores de referencia sociales. Una vez aceptada la publicación, la revista recuerda a sus autores lo recogido en la normativa mediante el envío de un correo electrónico, indicando la importancia que tiene para la revista la difusión de los artículos a través de los medios sociales del autor y de la revista, así como la participación activa en estos medios. 
Nuestro objetivo en esta investigación es detectar si en el caso de la revista española Comunicar, que, como se ha resaltado mantiene una clara política de uso y recomendación de los medios sociales para la difusión de sus publicaciones, los valores de las métricas tradicionales correlacionan en mayor medida con las altmétricas.

Esta revista se encuentra dentro de las áreas de Comunicación y Educación y posicionada en el primer cuartil de acuerdo con el JCR (Journal Citation Reports).

Para concluir este estudio, hemos elaborado una serie de recomendaciones a los investigadores a tener en cuenta a la hora de valorar estos datos y desde nuestra óptica de bibliotecarios universitarios de apoyo en los procesos de evaluación de la investigación (Tabla IV). En este sentido se debe ofrecer al investigador una respuesta sobre la utilidad de las altmétricas para que cuando pasen por procesos de evaluación, con indicadores científicos tradicionales, conozcan cómo han de ser tenidos en cuenta estos indicadores complementarios (González-Fernández-Villavicencio et al., 2015). En suma, si la inversión en tiempo que requieren se verá compensada por un crecimiento en sus expectativas de investigación y promoción académica.

Para este estudio nos hemos centrado en las siguientes preguntas de investigación:

- En el caso concreto de la revista Comunicar y a tenor de su política en medios sociales, ¿correlacionan las métricas tradicionales con las altmétricas en mayor medida que lo hacen en el resto de los estudios realizados hasta la fecha?

- En el caso de que no correlacionen, ¿cuáles podrían ser las posibles las causas?

- Desde la perspectiva de los bibliotecarios de apoyo a la investigación, ¿cuáles serían las recomendaciones que se podrían dar a los investigadores y editores para que consideren estas medidas o no en sus prácticas profesionales?

\section{LAS CORRELACIONES}

Para responder a la primera pregunta comenzamos revisando la bibliografía especializada sobre estudios de correlaciones entre métricas tradicionales y altmétricas, detectando que los resultados han sido poco significativos. Los autores han utilizado métodos estadísticos como regresión, correlación bivariada, coeficiente de correlación de Pearson o de Spearman, -el más utilizado-, llegando a convertirse en una constante en estos estudios y basando en ellos la validez y utilidad de las altmétricas (Haustein et al., 2015).

Para dar una idea de su magnitud, en el trabajo de Erdt et al. (2016), se recopilan correlaciones de Spearman realizadas en 25 trabajos que relacionan las altmétricas con los sistemas tradicionales de citas, Web of Science (WoS), Scopus y Google Scholar Citation (GSC).

En la correlación con citas recibidas, tan solo destaca Mendeley (Martín-Martín et al., 2016), así como en la cobertura de la producción científica indizada (Sugimoto et al., 2017; Robinson-García et al., 2014, p. 363; Suiter y Moulaison, 2015), convirtiéndose junto a Twitter (Hassan; Gillani, 2016), en prometedoras fuentes de datos sociales para las altmétricas.

Tampoco se observa una clara correlación al comparar los distintos indicadores altmétricos entre ellos (Peters et al., 2016); según los estudios, en algunos casos esta correlación ha sido fuerte y positiva y en otras moderada o incluso negativa (Haustein et al., 2015).

En trabajos que se han ocupado del cálculo de la correlación de estos indicadores desde la perspectiva de las disciplinas, la correlación de las altmétricas con los sistemas de recuentos de citas sigue siendo débil (Costas, Zahedi y Wouters, 2015b; Eysenbach, 2011; Haustein et al., 2015; Thelwall, 2016). En el área de Ciencias Sociales y Humanidades, Mendeley tiene una moderada correlación de Spearman con GSC (Bar-Ilan et al., 2013). Para el caso de Twitter, los artículos en Medicina General, Psicología y Ciencias Sociales son los que tienen mayor correlación con los recuentos de citas (Costas et al., 2015b).

Martín-Martín et al. (2016) muestran que los indicadores de GSC correlacionan muy bien con el resto de indicadores de publicación, cita o uso (tradicionales), pero no con los indicadores de conectividad (sociales). Sin embargo las métricas de conectividad social (seguidores) correlacionan entre sí, Mendeley, Twitter y ResearchGate. Para Tonia et al. (2016) estas métricas miden el valor añadido de una publicación científica y su difusión. Las métricas de Twitter por ejemplo, miden otra dimensión de la actividad social de los investigadores, favorecen que los artículos sean citados (Ortega, 2015), predicen con el Twimpact Factor artículos altamente citados en los tres primeros días de 
su publicación (Eysenbach, 2011) o capturan el impacto social de las publicaciones científicas con el alt-index de Hassan y Gillani (2016). Bornmann y Haunschild (2016a) proponen una metodología para normalizar las métricas de Twitter e investigan sobre el uso de $T$-factor de un investigador en comparación con el $H$-index.

Por tanto, Twitter y Mendeley parecen ser los más efectivos canales para medir el impacto social, aunque concretamente Twitter tenga una correlación más baja con las citas que Mendeley (Suiter y Moulaison, 2015). A ambos medios les siguen Facebook y Google+ (Hassan y Gillani, 2016).

\section{MATERIAL Y MÉTODOS}

En este estudio se han recopilado los 224 artículos que la revista Comunicar publicó desde octubre del año 2011 a julio de 2016, en concreto desde los números 37 a 48, para asegurar la coherencia con los datos altmétricos, ya que los rastreadores analizan con más seguridad las publicaciones a partir de 2011. Todos los datos se recogieron entre la segunda mitad de agosto y la primera de septiembre de 2016.

En primer lugar se han contabilizado las citas recibidas por estos artículos en WoS, Scopus y Google Scholar Citations (GSC). Para los datos altmétricos nos hemos basado en los ofrecidos por Altmetric.com y Snowball Metrics.

De Altmetric.com hemos tenido en cuenta el AAS, la posición de un artículo con respecto a otros -o percentil- y algunos de los datos que recopila de otras plataformas como son el número de tuiteros/tuits, lectores en Mendeley o el número de veces compartido en Facebook o en Google +, número de veces publicado en blogs, noticias, sitios de peer review, veces guardado en CiteULike y citas en Wikipedia.

De Snowball hemos seleccionado el SnowBall Field-Weighted Citation Impact, que compara la atención recibida por un artículo con otros de su categoría, según las citas recibidas en Scopus y el Social Activity Percentile, indicador que permite comparar la atención recibida por un artículo en redes sociales con respecto a otros de su categoría y que Snowball calcula a partir de los datos sobre Twitter y Facebook del recolector Altmetric.com. También hemos seleccionado los datos de Mendeley que SnowBall recolecta directamente de su API, y con el que conforma, junto con los datos de CiteULike, su Academic Activity Percentile, para comparar la atención académica recibida por un artículo con respecto a otros de su categoría. Estos dos últimos indicadores, Social y Academic Activity Percentile, se obtuvieron a mediados de 2016 y se han visto transformados con la adquisición de Plum Analytics por Elsevier, en 2017, de tal manera que, actualmente, el Social Activity Percentile ha sido sustituido por el indicador de Social Media y el Academic Activity Percentile se encuentra incluido en Captures, ambos de PlumX.

En el caso de Mendeley, se han obtenido también datos directamente de su interfaz, lo que nos ha permitido comparar la información que ofrecen los recolectores con la ofrecida por las fuentes y detectar las limitaciones que presentan los primeros, como la duplicidad de versiones en Mendeley no detectadas por Altmetric.com o Snowball.

La búsqueda de datos en Mendeley se ha realizado partiendo del título, en inglés y español, identificando las coincidencias en los primeros 20 resultados (primera página de resultados) y sumando los lectores de todas las versiones encontradas. Una misma versión encontrada en los resultados de las dos búsquedas, inglés y español, sólo se tiene en cuenta una vez.

En GSC se contabilizaron las citas del perfil de Comunicar y se completó con la búsqueda de cada artículo en español e inglés y la suma de las citas recibidas de artículos duplicados. El indicador por tanto es la fusión de las citas de diferentes versiones del artículo no repetido. No obstante, durante el estudio se ha identificado que GSC duplica algunas citas en la misma versión, hecho que se ha mantenido en este trabajo para no modificar la información pública que ofrece la propia herramienta.

Comprobando el score de Comunicar en Altmetric Explorer, se detectó que algunas veces se duplicaban los artículos al identificar dos IDs diferentes, DOI y Arxiv, y se trasladó el problema a Altmetric.com para su corrección, con efectos inmediatos.

Como se ha indicado, de Snowball se han utilizado los percentiles Social y Academic Activity, pero no todos los artículos recopilados en este estudio presentan ambos indicadores. Tampoco ofrece este recolector un score tipo $A A S$ y otros indicadores propios que podrían haberse considerado interesantes como Field-Weighted Citation Impact y Citation benchmarking que se refieren exclusivamente a citas tradicionales. 
Tras obtener todos los indicadores enumerados (Tabla I), se realiza el análisis estadístico de los datos, aplicando los coeficientes de correlación de Pearson y Spearman. Los resultados aportan la fuerza y el signo de la relación entre altmétricas y métricas basadas en citas y podemos establecer ciertas conclusiones con respecto a la revista Comunicar, que corroboran lo ya recogido en la bibliografía existente. A partir de ellas, aportamos una tabla de recomendaciones a los autores en el epígrafe de conclusiones (Tabla IV) sobre la necesidad o no de tener en cuenta estos indicadores y la forma de utilizarlos.

Los datos y las tablas de las correlaciones están publicados en Mendeley Data (González-Fernández-Villavicencio y Moya-Orozco, 2017).

\begin{tabular}{|l|l|}
\hline Fuente & Variables \\
\hline WoS & Citas \\
\hline Scopus & Citas \\
\hline Google Scholar Citations (GSC) & Citas \\
\hline Altmetric.com & $\begin{array}{l}\text { AAS (Altmetric Attention Score) } \\
\text { Tuiteros/tuits } \\
\text { Lectores en Mendeley } \\
\text { Veces compartido en Facebook } \\
\text { Veces compartido en Google }+ \\
\text { Post en blogs } \\
\text { Noticias } \\
\text { Sitios de peer review } \\
\text { Usuarios de CiteULike } \\
\text { Citas en Wikipedia }\end{array}$ \\
\hline Mendeley & $\begin{array}{l}\text { SnowBall Field-Weighted Citation Impact } \\
\text { Social Activity Percentile (ahora Social Media), que incluye datos de redes } \\
\text { sociales, principalmente Facebook y Twitter } \\
\text { Academic Activity Percentile (ahora Captures), que incluye lectores en } \\
\text { Mendeley y en CiteUlike }\end{array}$ \\
\hline
\end{tabular}

Tabla I. Resumen de proveedores y variables estudiadas. (Elaboración propia).

\section{ANÁLISIS Y RESULTADOS}

Las variables obtenidas se han dividido en los siguientes subconjuntos: relación entre métricas tradicionales y Mendeley y entre fuentes que proveen datos de Mendeley; relación entre valores altmétricos; relación entre altmétricas y métricas tradicionales; y un estudio final completo de correlaciones.

\subsection{Relación entre los recuentos de citas tradicionales y Mendeley}

El coeficiente de correlación de Pearson es fuerte y positivo entre los recuentos de citas que realizan los recursos WoS, Scopus y GSC, considerados en este estudio como grupo tradicional, pero más débil aunque moderado y positivo con los lectores de Mendeley a un nivel de significación de ,000 (Tabla II). 


\section{Correlaciones}

\begin{tabular}{|c|c|c|c|c|c|}
\hline & & Citas Scopus & Citas WoS & Citas GS & Mendeley \\
\hline \multirow[t]{3}{*}{ Citas Scopus } & Correlación de Pearson & 1 &, $830^{\text {*** }}$ &, $880^{* * *}$ &, $558^{* *}$ \\
\hline & Sig. (bilateral) & &, 000 &, 000 &, 000 \\
\hline & $\mathrm{N}$ & 224 & 224 & 224 & 220 \\
\hline \multirow[t]{3}{*}{ Citas WoS } & Correlación de Pearson &, $830^{* *}$ & 1 &, $747^{* *}$ &, $511^{* *}$ \\
\hline & Sig. (bilateral) &, 000 & &, 000 &, 000 \\
\hline & $\mathrm{N}$ & 224 & 224 & 224 & 220 \\
\hline \multirow[t]{3}{*}{ Citas GS } & Correlación de Pearson &, $880^{* *}$ &, $747^{* *}$ & 1 &, $607^{* *}$ \\
\hline & Sig. (bilateral) &, 000 &, 000 & &, 000 \\
\hline & $\mathrm{N}$ & 224 & 224 & 224 & 220 \\
\hline \multirow[t]{3}{*}{ Mendeley } & Correlación de Pearson &, $558^{* *}$ &, $511^{* *}$ &, $607^{* *}$ & 1 \\
\hline & Sig. (bilateral) &, 000 &, 000 &, 000 & \\
\hline & $\mathrm{N}$ & 220 & 220 & 220 & 220 \\
\hline
\end{tabular}

**. La correlación es significativa en el nivel 0,01 (bilateral).

Tabla II. Correlaciones de Pearson entre sistemas de citas tradicionales y Mendeley. (Elaboración propia).

Al estudiar las distintas fuentes que contemplan datos de Mendeley (Snowball, Altmetric.com y el propio Mendeley), la correlación es positiva y moderada entre la fuente y Mendeley de Snowball, pero débil aunque positiva con Mendeley de Altmetric.com (Tabla III). Como recogen otros autores, podría afirmarse que la debilidad de esa asociación se explica por la duplicidad y falta de fusión de las publicaciones por parte de Mendeley y porque algunos documentos con baja representación en el catálogo web de Mendeley no se encuentran disponibles vía API debido a los filtros de calidad que se les aplican (Tattersall, 2016, p. 89).

\section{Correlaciones}

\begin{tabular}{|c|c|c|c|c|}
\hline & & SB Mendeley & Mendeley & $\begin{array}{l}\text { Altmetric } \\
\text { Mendeley }\end{array}$ \\
\hline \multirow[t]{3}{*}{ SB Mendeley } & Correlación de Pearson & 1 &, $667^{* *}$ &, $351^{k * k}$ \\
\hline & Sig. (bilateral) & &, 000 &, 000 \\
\hline & $\mathrm{N}$ & 165 & 162 & 165 \\
\hline \multirow[t]{3}{*}{ Mendeley } & Correlación de Pearson &, $667^{* *}$ & 1 &, $461^{* *}$ \\
\hline & Sig. (bilateral) &, 000 & &, 000 \\
\hline & $\mathrm{N}$ & 162 & 221 & 221 \\
\hline \multirow[t]{3}{*}{ Altmetric Mendeley } & Correlación de Pearson &, $351^{* *}$ &, $461^{* *}$ & 1 \\
\hline & Sig. (bilateral) &, 000 &, 000 & \\
\hline & $\mathrm{N}$ & 165 & 221 & 224 \\
\hline
\end{tabular}

**. La correlación es significativa en el nivel 0,01 (bilateral).

Tabla III. Correlaciones de Pearson entre distintas fuentes que ofrecen datos de Mendeley. (Elaboración propia).

\subsection{Relación entre valores altmétricos}

Los percentiles de los sistemas de citas altmétricas, Percentil de Altmetric.com y Social Activity Percentile de Snowball correlacionan de forma positiva pero débil y con poco nivel de significación. Sin embargo, los percentiles de Actividad social y Actividad académica de Snowball, correlacionan de forma moderada y positiva $(, 567)$. 
Las variables de Altmetric.com -Facebook, Tuits, Mendeley, Google+, blogs, Noticias, sitios de peer review, CiteUlike y Wikipedia- correlacionan entre sí de forma débil y en algunos casos con signo negativo. Las citas en Facebook y en tuits son las únicas variables que presentan una correlación más significativa (,344), positiva y con nivel de significación ,000. Las variables de Wikipedia presentan una relación poco significativa y negativa con las demás variables excepto con Mendeley $(, 145)$. En general las correlaciones son muy débiles y en gran medida negativas, indicando una relación inversa entre variables. El mayor problema detectado es la escasez de datos en medios sociales de estos artículos, exceptuando Facebook y Twitter, sin duda relacionado con el poco generalizado uso de los medios sociales por parte de los investigadores, al menos en generaciones de más edad.

\subsection{Relación entre altmétricas y métricas tradicionales}

Entre los sistemas de citas tradicionales y el score de Altmetric.com, la relación es positiva pero muy débil, score de Altmetric.com con Scopus ,054; con WoS ,062; con GSC ,012.

Entre los sistemas de citas tradicionales, Twitter de Altmetric.com y Twitter en Snowball, las correlaciones son poco significativas y negativas, la evolución es inversa en ambos casos.

Entre los sistemas de citas tradicionales y Facebook de Altmetric.com y Facebook de Snowball, la correlación es muy débil en ambos casos, de signo negativo para Facebook de Altmetric.com y positivo para las variables de Facebook de Snowball.

\subsection{Estudio de las correlaciones bivariadas de Pearson y rho de Spearman entre todas las variables estudiadas}

Se confirma el alto nivel de correlación entre las variables de las citas tradicionales incluyendo GSC y Mendeley, tanto con el coeficiente de correlación de Pearson como rho de Spearman.

Muy débil nivel de correlación de las métricas tradicionales con las altmétricas, tanto con el coeficiente de Pearson $^{2}$ como el de rho de Spearman. Entre las variables altmétricas, solo correlacionan fuerte y positivamente los valores de Altmetric.com, score y tuits de Altmetric.com (,840), y estas mismas con el Percentil de Altmetric.com correlacionan de forma moderada $(, 694$ y ,625), positiva y significativa.

Con el coeficiente rho de Spearman ${ }^{3}$ correlacionan más variables, se fortalece la relación entre score y tuits de Altmetric.com $(, 897)$ y de forma moderada Facebook y Mendeley de Altmetric.com.

\section{LIMITACIONES DEL ESTUDIO}

El estudio llevado a cabo se centra en los datos proporcionados por las métricas tradicionales y altmétricas sobre la revista Comunicar, que representa un caso excepcional de uso de los medios sociales para la difusión de sus publicaciones y por lo tanto podría esperarse mayor incidencia de las altmétricas. No obstante sería interesante realizar una comparativa entre varias revistas de la misma área o por disciplinas, con distintos modelos de planes de marketing en medios sociales, así como la ampliación de las series temporales de datos obtenidos, para comparar resultados y relaciones y establecer tendencias futuras.

Algo evidente en este estudio ha sido la escasez de datos altmétricos que nos hemos encontrado, tan sólo el $65 \%$ de los artículos presentaba datos en el AAS de Altmetric.com. Se trata de un dato relevante para una revista con presencia activa en medios sociales. Sin embargo consideramos que se trata de una actividad en alza, donde las nuevas generaciones están instaladas y el número de datos altmétricos aumentará con los años, permitiendo extraer conclusiones más determinantes y ligadas a sus planes de marketing.

A la escasez de datos se une la falta de consistencia. De los 224 artículos estudiados, 59 no contaban con datos de Snowball, entre ellos los publicados durante el 2016 y un número completo de 2015. Los datos existentes no hacían referencia a todas las categorías que incluye la revista (Educación, Comunicación y Estudios Culturales), variando según el artículo. Hemos detectado también diferencias en la recopilación de datos con respecto a los que ofrece Altmetric.com, especialmente en relación a Twitter, Facebook y otras redes sociales, a pesar de que provienen de Altmetric.com.

En el caso de los datos sobre Mendeley, la fuente de Snowball es directamente la API del gestor social, lo que podría explicar diferencias en los datos de ambos agregadores por su forma de proceder y configurar sus sistemas de rastreo. Según NISO (2016) los datos relativos a los lectores totales en Mendeley y los lectores por estatus académico 
y disciplinas, en el mismo periodo de tiempo, pueden ser diferentes en el catálogo web de Mendeley y en las API ya que no se calculan de forma simultánea. Para el mismo documento podemos encontrar un número de lectores diferente si se han usado metadatos distintos, -como DOI o título del documento-, y diversos métodos de recuperación, -ya sea catálogo web o API-, aunque sólo en el caso de aquellos duplicados en los que uno tiene el identificador usado en la búsqueda y el otro no lo tiene. Así lo hemos comprobado en este estudio al comparar los datos extraídos de forma manual con los procedentes de los servicios altmétricos.

Por último, hubiera sido interesante contabilizar las descargas de estos artículos durante el periodo de toma de datos.

Para un estudio posterior, sería interesante ampliar el rango de datos en un periodo temporal mayor y comparar estos datos con los altmétricos que en estos momentos está proveyendo PlumX para Scopus.

\section{CONCLUSIONES}

Respondiendo a nuestras preguntas de investigación, podemos concluir que los resultados de los estudios de correlaciones en la revista Comunicar, a pesar de llevar adelante un cuidado plan de marketing en medios sociales, reflejan la misma situación que en los estudios de referencia, es decir, los valores son significativos entre las métricas tradicionales pero no correlacionan significativamente con todos los valores altmétricos. Para esta revista podemos indicar que el nivel de correlación que hemos encontrado, entre los sistemas de citas tradicionales y Mendeley es bastante elevado así como con Twitter, aunque en menor medida, y que posiblemente seguirá aumentando a medida que se generalice el uso de los medios sociales por los investigadores. Sin lugar a dudas, la implantación de un plan de marketing en medios sociales y su continuidad en el tiempo, podrán contribuir a una mayor relación de dependencia entre las métricas tradicionales y las altmétricas.

En cuanto a las correlaciones entre las altmétricas, nos ha sorprendido la ausencia o debilidad de la relación entre ellas. La ausencia de datos ha dificultado el establecimiento de correlaciones más fiables. La falta de correlación afecta incluso a las variables del mismo recurso procedentes de distintas fuentes, como es el caso de Mendeley, problema ya documentado en la última publicación de NISO (2016). Este problema se explica por la duplicidad y falta de fusión de los documentos al aplicarse filtros de calidad de los contenidos, pero esta restricción desaparecerá cuando los datos aportados por los lectores en Mendeley sean validados con los datos aportados desde Scopus (Tattersall, 2016, p. 89). No obstante creemos que esta deficiencia contribuye a entender cómo deben usar los investigadores estos sistemas.

Teniendo en cuenta que Mendeley y Twitter son las redes que más correlacionan, la presencia en ambas es esencial para incrementar la visibilidad y la difusión de las publicaciones de los investigadores, sobre todo si obedecen a una cuidada planificación o se utilizan herramientas específicas para planificar esa difusión como puede ser Kudos, que está siendo recomendada por gran parte de las revistas científicas (González-Fernández-Villavicencio, 2017). Una presencia activa y sobre todo planificada en Twitter, así como la correcta incorporación de las publicaciones en Mendeley, pueden contribuir a un incremento de lectores de los artículos que se traduzca a posteriori en más citas además de un mayor impacto económico, social, cultural o político de los resultados de investigación.

Otra consideración a tener en cuenta es la condición de acceso abierto y de campo específico de la revista Comunicar. Para Tattersall (2016, p. 93), ambas condiciones favorecen las citas tanto en sistemas tradicionales como altmétricos. Comprobamos aquí la importancia del acceso abierto tanto para los investigadores como para los propios editores de publicaciones científicas. El modelo de Acceso Abierto facilita la recuperación de la información, aún más cuando los indicadores tradicionales y altmétricos de la revista están tan consolidados como en el caso de la revista Comunicar. A finales de 2017 Clarivate Analytics está potenciando el acceso y la difusión de las publicaciones en Acceso Abierto en su producto Web of Science con la colaboración del proveedor de datos altmétricos Impactstory.

Se han encontrado algunas coincidencias cualitativas. No creemos que sea casual que uno de los artículos más citados y que a la vez obtenga valores más altos en las altmétricas sea el de Torres-Salinas, dedicado también al tema de las altmétricas. Creemos que estos resultados se deben tanto al uso de las redes sociales de sus autores como a la importancia y el interés que despierta este tema por su transversalidad, en otras disciplinas más allá del alcance de la propia revista -Biblioteconomía y Documentación-, por ejemplo.

Por otro lado, las nuevas herramientas vinculadas a la difusión de las publicaciones científicas están fomentando el uso de estos medios. Un estudio de $2016^{4}$ asegura que los artículos explicados en Kudos obtienen un promedio de un 23\% más de descargas (más difusión pero no más citas de otros investigadores). Las redes sociales académicas, la 
formación específica que ofrecen las bibliotecas universitarias o la aparición creciente de datos altmétricos en revistas científicas, en los catálogos de bibliotecas, bases de datos (como puede ser la reciente adquisición de Plum Analytics por Elsevier y la presencia de sus datos en la base de datos Scopus), y repositorios institucionales, contribuyen a una mayor difusión de las altmétricas por parte de los investigadores, unido a un mayor entendimiento de su significado y transparencia como indica el último informe de la NISO (2016).

En la Tabla IV se aporta un resumen de las recomendaciones sobre el uso de las altmétricas para investigadores y editores, que se han ido presentando a lo largo del artículo.

1. Los investigadores deben tener en cuenta las altmetricas ya que ayudan a completar el alcance de la investigación más allá de los canales tradicionales, amplían la audiencia no académica, ofrecen indicadores de un impacto en tiempo real y son públicamente accesibles.

2. Cada vez están más presentes en recursos bibliográficos tradicionales como PlumX en Scopus.

3. Los investigadores deben planificar su presencia en medios sociales para conseguir una mayor difusión de su producción científica y obtener reconocimiento y oportunidades de negocio.

4. Las editoriales deben establecer un plan de marketing en medios sociales que les ayude a alcanzar los objetivos de visibilidad de sus publicaciones.

5. Dada la mayor correlación con las citas tradicionales, se recomienda que Mendeley y Twitter sean los medios sociales que no deben faltar en la planificación de la actividad en medios sociales tanto para investigadores como editores.

6. Los investigadores deben incorporar sus publicaciones correctamente en Mendeley a través del DOI para evitar versiones duplicadas y no congruentes, así como comprobar que no existen duplicados de sus publicaciones.

7. Los investigadores deben contar con una presencia activa y sobre todo planificada en Twitter.

8. El uso de herramientas de gestión de medios sociales, como Kudos, facilita esta tarea a los investigadores.

9. Publicar en revistas en Acceso Abierto favorece la visibilidad y la atención en medios sociales.

10. Creemos que los ámbitos de publicación transversales favorecen el incremento de la visibilidad y los resultados altmétricos de autores y publicaciones.

\section{Tabla IV. Diez recomendaciones sobre uso de las altmétricas. (Elaboración propia).}

Con respecto a la revista Comunicar, creemos que los pasos dados hacia la difusión de sus publicaciones han sido estratégicos. La petición a los autores de difundir sus publicaciones en la revista a través de los distintos medios a su disposición es básico para obtener buenos indicadores altmétricos, si bien es necesario que los investigadores asuman más responsabilidad en esta actividad e incorporen la difusión como una fase más de su trabajo de publicación. De esta forma lo indicaban Cabezas-Clavijo, Torres Salinas y Delgado-López-Cózar (2009) cuando hablaban del ciclo estratégico de difusión de la investigación. Nosotros añadimos que esa actividad debe formar parte de la rutina del investigador y en el caso de las revistas, debe ser planificada en un plan de marketing en medios sociales, que forme parte de la actividad habitual del equipo editorial y afecte también a los agentes de la producción científica, siempre bajo el paraguas de una política corporativa de uso de los medios sociales.

\section{NOTAS}

${ }^{1}$ Información en la revista $<$ https://goo.gl/DxPBIK $>$.

${ }^{2}$ Dirección URL de las correlaciones de Pearson $<$ https://goo.gl/J6NvwF $>$.

${ }^{3}$ Dirección URL de la tabla de correlaciones de rho de Spearman $<$ https://goo.gl/WqoBXN $>$.

${ }^{4}$ Resultados del estudio $<$ https://goo.gl/53lqqm>. 


\section{BIBLIOGRAFÍA}

BAR-ILAN, J. et al. Altmetrics: Present and Future - Panel. Proceedings of the American Society for Information Science and Technology, 2013, vol. 50, $\mathrm{n}^{\circ}$ 1, p. 1-4, doi:10.1002/meet.14505001013.

BORNMANN, L. Do altmetrics point to the broader impact of research? An overview of benefits and disadvantages of altmetrics. Journal of Informetrics, 2014, vol. 8, no 4, p. 895-903, doi:10.1016/j.joi.2014.09.005.

BORNMANN, L. y HAUNSCHILD, R. How to normalize Twitter counts? A first attempt based on journals in the Twitter Index. Scientometrics, 2016, vol. 107, no 3, p. 1-18, doi:10.1007/s11192-016-1893-6.

BORNMANN, L. y HAUNSCHILD, R. Does evaluative scientometrics lose its main focus on scientific quality by the new orientation towards societal impact? Scientometrics, 2016, vol. 110, nº 2, p. 937-943, doi:10.1007/s11192016-2200-2.

CABEZAS-CLAVIJO, A.; TORRES-SALINAS, D. y DELGADO-LÓPEZ-CÓZAR, E. Ciencia 2.0: catálogo de herramientas e implicaciones para la actividad investigadora. El profesional de la información, 2009, vol. 18, $\mathrm{n}^{\mathrm{0}} 1$, p. 75-82, doi:10.3145/epi.2009.ene.10.

COSTAS, R.; ZAHEDI, Z. y WOUTERS, P. Do "altmetrics" correlate with citations? Extensive comparison of altmetric indicators with citations from a multidisciplinary perspective. Journal of the Association for Information Science and Technology, 2015a, vol. 66, $\mathrm{n}^{\mathrm{o}}$ 10, p. 2003-2019, doi:10.1002/asi.23309.

COSTAS, R.; ZAHEDI, Z. y WOUTERS, P. The thematic orientation of publications mentioned on social media: large- scale disciplinary comparison of social media metrics with citations. Aslib Journal of Information Management, 2015b, vol. 67, no 3, p. 260-288.

ERDT, M.; NAGARAJAN, A.; SIN, S.C.J. y THENG, Y.L. Altmetrics: an analysis of the state-of-the-art in measuring research impact on social media. Scientometrics, 2016, vol. 109, nº 2, p. 1-50, doi:10.1007/s11192-016-2077-0.

EYSENBACH, G. Can Tweets Predict Citations? Metrics of Social Impact Based on Twitter and Correlation with Traditional Metrics of Scientific Impact. Journal of Medical Internet Research, 2011, vol. 13, no 4, p. e123. doi:10.2196/jmir.2012.

GONZÁLEZ-FERNÁNDEZ-VILLAVICENCIO, N.; CALDERÓN-REHECHO, A.; DOMÍNGUEZ-AROCA, M.-I. y GARCÍA-HERNÁNDEZ, P. ¿Qué papel juegan los bibliotecarios en las altmetrics? Anales de documentación, 2015, vol. 18 , no 2, p. 1-19, doi:10.6018/analesdoc.18.2.222641.

GONZÁLEZ-FERNÁNDEZ-VILLAVICENCIO, N. Una sola métrica no cuenta toda la historia de la producción científica. I. Visibilidad. Revista ORL, 2016, vol. 7, nº 3, p. 143-149, doi:10.14201/orl201673.14444.

GONZÁLEZ-FERNÁNDEZ-VILLAVICENCIO, N. y MOYA-OROZCO, V.M. ¿Las correlaciones altmetricas/metricas tradicionales se ven afectadas por los planes de marketing en medios sociales de las revistas? el caso de la revista Comunicar. Disponible en: Mendeley Data, 2017, vol. 1, doi:10.17632/78hpjhhzbd.3

GONZÁLEZ-FERNÁNDEZ-VILLAVICENCIO, N. Altmetrics y las políticas de difusión en medios sociales de las revistas científicas. En $7^{a}$ Conferencia internacional sobre revistas de ciencias sociales y humanidades Cuenca, 45 mayo, 2017 [en línea]. Disponible en: <http://thinkepi.net/notas/crecs_2017/MesaMarketing_Gonzalez.pdf>. [Consulta: 29 de agosto de 2017].

HASSAN, Fs.-U. y GILLANI, U.A. Altmetrics of «altmetrics» using Google Scholar, Twitter, Mendeley, Facebook, Google-plus, CiteULike, Blogs and Wiki. arXiv preprint 2016 [en línea]. Disponible en: $<$ https://arxiv.org/abs/1603.07992>. [Consulta: 29 de agosto de 2017].

HAUSTEIN, S.; SUGIMOTO, C.R. y LARIVIÈRE, V. Guest Editorial: Social media in scholarly communication. Aslib Journal of Information Management, 2015, vol. 67, $\mathrm{n}^{\mathrm{O}}$ 3, p. 1-7.

KONKIEL, S. Altmetrics: diversifying the understanding of influential scholarship. Palgrave Communications, 2016, vol. 2, p. 16057. doi:10.1057/palcomms.2016.57.

MARTÍN-MARTÍN, A.; ORDUÑA-MALEA, E.; AYLLÓN, J.M. y DELGADO LÓPEZ-CÓZAR, E. The counting house, measuring those who count: Presence of Bibliometrics, Scientometrics, Informetrics, Webometrics and Altmetrics in Google Scholar Citations, ResearcherID, ResearchGate, Mendeley, \& Twitter. EC3 Working Papers, 2016, vol. 21, $\mathrm{n}^{\mathrm{o}}$ enero, doi:10.13140/rg.2.1.4814.4402.

NATIONAL INFORMATION STANDARDS ORGANIZATION (NISO). Outputs of the NISO Alternative Assessment Metrics Project RP 25-2016, 2016.

ORTEGA, J.L. Relationship between altmetric and bibliometric indicators across academic social sites: The case of CSIC's members. Journal of Informetrics, 2015, vol. 9, no 1, p. 39-49, doi:10.1016/j.joi.2014.11.004.

PATTON, R.M.; STAHL, C.G. y WELLS, J.C. Measuring Scientific Impact Beyond Citation Counts. D-Lib Magazine, 2016, vol. 22, nº 9/10, p. 1-10, doi:10.1045/september2016-patton.

GONZÁLEZ FERNÁNDEZ-VILLAVICENCIO, N. y MOYA OROZCO, V.M. ¿Las correlaciones altmétricas/métricas tradicionales se ven afectadas por los planes de marketing en medios sociales de las revistas? El caso de la revista Comunicar. Anales de Documentación, 2018, vol. 21, nº 1. Disponible en: http://dx.doi.org/10.6018/analesdoc.21.1.303221. 
PETERS, I. et al. Research data explored: an extended analysis of citations and altmetrics. Scientometrics, 2016 , vol. 107, $\mathrm{n}^{\mathrm{o}}$ febrero, p. 723-744, doi:10.1007/s11192-016-1887-4.

PIWOWAR, H. Altmetrics: Value all research products. Nature, 2013, vol. 493, doi:10.1038/493159a.

PRIEM, J.; TARABORELli, D.; GROTH, P. y NEYLON, C. Altmetrics: A manifesto [en línea]. Disponible en: $<$ http://altmetrics.org/manifesto/>. [Consulta: 29 de agosto de 2017].

ROBINSON-GARCÍA, N.; TORRES-SALINAS, D.; ZAHEDI, Z. y COSTAS, R. New data, new possibilities: Exploring the insides of. El profesional de la información, 2014, vol. 23, $\mathrm{n}^{\mathrm{o}}$ 4, p. 359-366, doi:10.3145/epi.2014.jul.03.

SUGIMOTO, C.R.; WORK, S.; LARIVIÈRE, V. y HAUSTEIN, S. Scholarly use of social media and altmetrics: a review of the literature. Journal of the Association for Information Science and Technology [en línea], 2017, vol. 68, $\mathrm{n}^{\circ}$ 9, p. 2037-2062. Disponible en: <http://arxiv.org/abs/1608.08112>. [Consulta: 29 de agosto de 2017].

SUITER, A.M. y MOULAISON, H.L. Supporting Scholars: An Analysis of Academic Library Websites' Documentation on Metrics and Impact. The Journal of Academic Librarianship, 2015, vol. 41, $\mathrm{n}^{\circ}$ 6, p. 814-820, doi:10.1016/j.acalib.2015.09.004.

TATTERSAL, A. (ed.). Altmetrics. A practical guide for librarians, researchers and academics. London: Facet Publishing, 2016.

THELWALL, M. Interpreting correlations between citation counts and other indicators. Scientometrics, 2016 , vol. 108, no 1, p. 337-347, doi:10.1007/s11192-016-1973-7.

TONIA, T. et al. If I tweet will you cite? The effect of social media exposure of articles on downloads and citations. International Journal of Public Health, 2016, vol. 61, no 4, p. 513-520, doi:10.1007/s00038-016-0831-y.

TORRES-SALINAS, D.; CABEZAS-CLAVIJO, A.; JIMÉNEZ-CONTRERAS, E. y TORRES, D. Altmetrics: nuevos indicadores para la comunicación científica en la web 2.0. Comunicar. Revista científica de educomunicación, vol. 41, $\mathrm{n}^{\mathrm{o}}$ XXI, p. 53-60, doi:10.3916/C41-2013-05. 